\title{
Londres : une géographie du renouveau des tours
}

London: A geography of skyscrapers regeneration

London: eine Geografie der Hochhäuserrenovierung

\section{Jean-Philippe Hugron}

\section{(2) OpenEdition}

\section{Journals}

Édition électronique

URL : http://journals.openedition.org/rge/1085

DOI : $10.4000 /$ rge. 1085

ISSN : 2108-6478

\section{Éditeur}

Association des géographes de l'Est

Édition imprimée

Date de publication : 1 janvier 2008

ISSN : 0035-3213

\section{Référence électronique}

Jean-Philippe Hugron, «Londres : une géographie du renouveau des tours », Revue Géographique de

l'Est [En ligne], vol. 48 / 1-2 | 2008, mis en ligne le 09 octobre 2011, consulté le 08 septembre 2020.

URL : http://journals.openedition.org/rge/1085 ; DOI : https://doi.org/10.4000/rge.1085

Ce document a été généré automatiquement le 8 septembre 2020.

Tous droits réservés 


\title{
Londres : une géographie du renouveau des tours
}

\author{
London: A geography of skyscrapers regeneration \\ London: eine Geografie der Hochhäuserrenovierung
}

Jean-Philippe Hugron

\section{Introduction}

1 Millbank Tower, Empress State Building, Euston Tower, le paysage urbain de Londres est scandé de tours aux styles différents, de l'art déco, dont se réclame le premier gratte-ciel de la capitale britannique, Adelaïde House (1925), à l'high-tech en passant par le modernisme et le postmodernisme. Leur coprésence démontre que quelle que soit l'époque, l'architecture verticale s'est inscrite de façon plus ou moins importante dans la ligne d'horizon. Elle se pense aujourd'hui de façon magistrale, et le skyline londonien s'enrichira bientôt d'édifices à la hauteur inédite et aux formes audacieuses. La City sera dominée par les 280 mètres de Bishopsgate Tower, Southwark et la Tamise par les 310 mètres de la London Bridge Tower. Le marché de l'immobilier en plein essor signe un engouement renouvelé pour le fait vertical dans la capitale britannique après une période d'endiguement qui a vu naître Canary Wharf.

2 Inscrites dans une stratégie spatiale conduite par le gouvernement britannique et la Greater London Authority, les tours servent les vastes projets de régénération comme l'image de la ville qui confirme ainsi à travers quelques projets signés par les starchitectes du moment son rôle de "world city ». Parallèlement, nombreuses tours héritées du modernisme ou du brutalisme architectural, sont transformées ou modernisées quand elles ne disparaissent pas, détruites, pour être remplacées par des édifices plus grands et plus adaptés à l'actuelle demande.

3 Si les tours constituent un type d'architecture consensuellement défini, l'apparente unité que recouvrirait le thème de l'architecture verticale est au regard de projets et de constructions en cours à Londres au cœur de tensions certaines entre reconversion, 
rénovation, réhabilitation, et patrimonialisation. Aussi une géographie des tours, alors que la discipline n'analyse que peu ou prou ces objets, ne peut dès lors faire l'économie d'une typologie.

Dans ce dessein nous reviendrons sur une série de plans d'urbanisme ayant influencé le développement de l'architecture verticale, et au premier rang desquels l'actuel London Plan et nous envisagerons les divers processus qui portent actuellement la construction de tours.

\section{Des tours, de l'attentisme à l'incitation ou le renouveau de la verticalité à Londres.}

Depuis l'avènement des gratte-ciel aux Etats-Unis au XIX ${ }^{\mathrm{e}}$ siècle et leur introduction en Europe au début du $\mathrm{XX}^{\mathrm{e}}$, le paysage urbain de Londres a été ponctué de tours (cf. fig. 1). Aussi, comment la construction de tours a-t-elle été et reste autorisée et quelles en étaient et sont les motivations ? Du débat d'idées, de la proposition à la construction d'édifices, les faits urbains comme la législation, témoignent de positionnements circonstanciels. La dernière évolution en date s'illustre par une politique incitative qui trouve pour contexte l'émergence de quelques projets. Tout concourt désormais au renouveau de la verticalité à Londres.

Figure 1 : carte les tours de Londres, un état des lieux

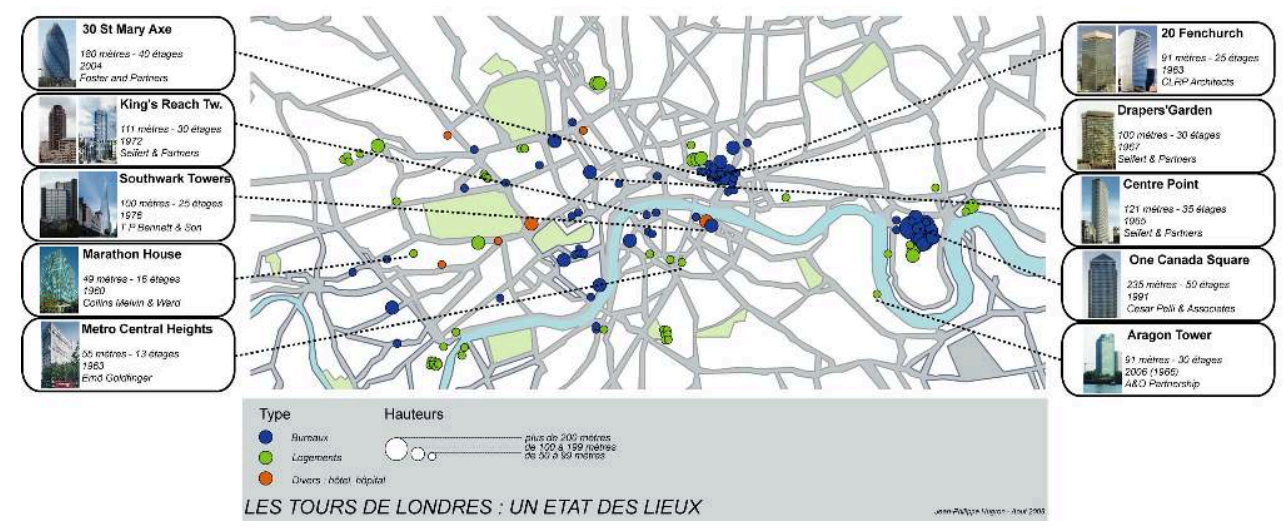

\section{A. Réglementation et faits urbains}

6 «Dans la City, l'obscurité s'est dissipée et le jour entre à flots par les vitres propres et les glaces aveuglantes des gratte-ciel. [...] La voici, légère, métallique, chromée, déjà adaptée à demain " (Morand, 1962, p. 486). Après avoir consacré un premier ouvrage à la capitale britannique, Paul Morand se voit offrir par l'éditeur en 1962 l'opportunité d'actualiser son écrit. Insufflant un esprit nouveau à ses lignes, il souhaite alors témoigner d' " une ville nouvelle, [d'] une Londres blanche [qui] monte dans le ciel » (Morand, 1962, p. 496). Le modernisme d'après-guerre scande le paysage londonien de tours. Elles sont héritées d'une politique incitative de reconstruction des édifices détruits lors des bombardements de la Seconde Guerre Mondiale. Les conservateurs ont voté par deux fois en 1947 et 1953 une augmentation de 10 \% des surfaces à reconstruire dans le cadre $\mathrm{du}$ Town and Country Planning Act. Ce dispositif trouve ses limites alors qu'il est impossible de dépasser le plafond des hauteurs prévu par le London Building Act fixé à 
30 mètres voire 25 mètres pour les édifices ayant une base supérieure à $930 \mathrm{~m}^{2}$. Cette limitation fixée en 1894 répond aux exigences sécuritaires de la protection contre l'incendie, les échelles de pompier ne pouvant atteindre des hauteurs supérieures.

La loi est révisée en 1956, et il revient à partir de cette date, au London County Council (LCC) d'accorder ou non un dépassement des hauteurs au cas par cas, au regard des qualités de chaque projet selon huit critères : l'intégration visuelle, le positionnement, le site, l'ombre portée, la physionomie du quartier, les impacts paysagers depuis la Tamise ou depuis les espaces ouverts, la qualité architecturale, l'intégration nocturne ${ }^{1}$ (Short, 2005).

8 En 1959 débute le projet de la plus haute tour jamais réalisée dans la capitale, Vickers Tower. Elle atteint la hauteur inégalée de 118 mètres. C'est alors que de nombreux projets de tours sont approuvés par le LCC qui en retour dispose des espaces libérés par ces constructions verticales pour réaliser au sol espaces verts et infrastructures viaires, nécessaires au bon essor du trafic. Les préceptes fonctionnalistes sont alors de rigueur.

En 1963, Portland House et le London Hilton érigent leur profil au dessus de Hyde Park et de St James Park. Ces espaces sacro-saints jusqu'alors préservés voient apparaître par delà la cime des arbres la confiance en un monde moderne. Buckingham Palace s'implique et dénonce l'intrusion de ces réalisations dans le paysage. La famille royale affirme sa position alors qu'une tour est proposée à Haymarket; la New Zeland House doit atteindre 95 mètres. Le Prince Philip intervient et le projet est alors réduit à 69 mètres.

En 1969, le Greater London Council (GLC) remplace le LCC. La réglementation en vigueur relative aux immeubles de grande hauteur est maintenue et complétée par un plan délimitant trois zones: des zones inappropriées aux immeubles de grande hauteur, des zones sensibles aux impacts visuels, des zones où la construction verticale pourrait être autorisée. Jusqu'en 1980 tous les projets d'immeuble supérieur à $45 \mathrm{~m}$ étaient du ressort du GLC.

11 A partir de 1986, date de dissolution du GLC, tout projet de tour est soumis au gouvernement en la personne du secrétaire d'Etat à l'environnement. Ces années marquent une période d'endiguement (Simon, 1996). L'architecture verticale controversée se trouve alors reléguée à l'est de Londres. Les rares réalisations sont alors cantonnées à Canary Wharf, la friche industrialo-portuaire qui ambitionne de devenir une seconde City, un "Wall Street on water » selon le plan de Skidmore, Owings et Merrill. L'aménagement est confié à une Urban Development Corporation (UDC), établissement dont les moyens financiers sont essentiellement d'origine privée et qui gère de manière dérogatoire les droits du sol. De fait le Borough of Tower Hamlets est dessaisi de ces prérogatives en termes d'aménagement. Le périmètre n'est alors plus soumis aux restrictions de hauteurs.

Parallèlement, le secrétaire d'Etat à l'environnement ordonne en août 1989 un rapport sur «la protection des vues importantes ». L'étude envisage une réglementation qui, votée en octobre 1991, exige la mise en place de dix couloirs de protection des vues. Ce dispositif est une autre conséquence de l'endiguement qui trouve alors une expression spatiale différente. Les interstices et autres espaces en dehors de ces corridors sont à même de recevoir, isolément, quelques constructions verticales.

13 Cette politique dite littéralement de "containment " est rapidement remise en cause alors qu'en 1996, en lieu et place du Baltic Exchange Building, endommagé en 1992 lors 
d'un attentat revendiqué par l'IRA, est proposée l'érection de la plus haute tour d'Europe, la London Millennium Tower. L'architecture de Sir Norman Foster devait dominer la City du haut de ces 386 mètres. English Heritage comme BAA, l'opérateur privé de l'aéroport d'Heathrow, situé à 25 kilomètres à l'ouest de la ville, s'opposèrent au projet. Deux ans plus tard, Calatrava proposa pour un autre site de la City une tour de 200 mètres. Portant atteinte aux vues depuis Saint-Paul, elle fut elle aussi ajournée. La juxtaposition des couloirs aériens et des perspectives visuelles protégées s'offre comme autant de contraintes et d'arguments témoignant du rejet significatif des tours malgré un cadre juridique autorisant leur construction.

Dans ce contexte, en 1998, Tony Blair, alors Premier Ministre, charge l'architecte Lord Richard Rogers ${ }^{2}$ de former l'Urban Task Force ${ }^{3}$ afin d'identifier les causes du déclin urbain en Angleterre et d'en proposer un diagnostic. En plus de recommandations sociales, environnementales et économiques, le rapport insiste sur la lutte contre l'étalement urbain et la nécessaire densification de l'espace sans pour autant évoquer l'urbanisme vertical ni même proposer l'érection de tours.

15 En 1999, après référendum, vote au parlement, et assentiment royal, le Greater London Authority Act est adopté. Par cette loi est formée une collectivité locale dont l'objectif est de coordonner les politiques d'aménagement et de réaliser en conséquence un plan stratégique à l'échelle du Grand Londres.

En 2000, Ken Livingstone accède au nouveau poste de maire de Londres et accorde à Lord Rogers le titre de conseiller en chef en architecture et en urbanisme. Ce dernier propose les éléments d'une "renaissance urbaine » reprenant in extenso les points élaborés par l'Urban Task Force. De son coté Ken Livingstone affirme sa position en faveur des tours ${ }^{4}$ (GLA, 2000). L'élaboration du London Plan débute par une consultation, dont le maire a l'initiative, «Towards the London Plan » où, de nouveau, il réaffirme son point de vue (GLA, 2001c, p.52). Suite à l'examen des réponses à la consultation engagée, le GLA répond favorablement en soutenant officiellement l'ambition du maire (GLA, 2002, p. 8). Il s'ensuit un rapport demandé au bureau d'étude DEGW (2002) qui précise le rôle des immeubles de grandes hauteurs. Les conclusions apportées, qui n'engagent pas le GLA, seront réutilisées afin d'élaborer le London Plan. Publié le 10 février 2004, le document accorde plusieurs paragraphes aux immeubles de grande hauteur. Aujourd'hui en vigueur, le plan témoigne d'une évolution majeure. Si les premières politiques étaient seulement permissives, autorisant ponctuellement la construction de tours, ce dernier plan est explicitement incitatif et consacre alors le renouveau de la verticalité à Londres.

\section{B. Urban Task Force et la renaissance des tours}

17 Parmi les premières politiques autorisant l'édification d'immeubles de grande hauteur, aucune ne prenait parti pour un urbanisme vertical. Cette réserve s'exprime alors que les instruments juridiques, tels la mise en place de zones inappropriées aux tours comme de couloirs de vue se multiplient et visent à contenir la forte demande de bureaux. A l'inverse, les tours sont actuellement vécues comme une solution aux problèmes urbains, notamment environnementaux. C'est alors non plus la ville qui compose avec la demande, mais la ville qui propose une réflexion en envisageant l'urbanisme vertical comme réponse. 

Buildings and Strategic Views in London est réalisé à la demande du gouvernement par le London Planning Advisory Committee dans le cadre de l'élaboration du Strategic Guidance for London Planning Authorities. Cette recherche affirme que «les tours ne sont pas une nécessité économique mais une question d'image, d'esthétique, de transport public " (GLA, 2001a, p. 6). urbaine " consacrant le rôle de la densité ainsi que celui de la qualité architecturale (Urban Task Force, 1999). Toutefois les mots de tour ou d'immeuble de grande hauteur ne sont jamais utilisés. A bon escient puisque la densification n'implique pas systématiquement la verticalisation. Lord Rogers, qui a rejoint l'équipe municipale en 2000, explique que son rôle de conseiller lui implique de tenir informé le maire des principes de l'Urban Task Force et de les rendre applicables à Londres (Rogers, 2000, p.4). Il apporte en plus une vision personnelle, et dans ce contexte, Lord Rogers n'hésite plus à utiliser le mot tour (tall building)5. Il s'engage à exprimer son opinion quant à la skyline londonienne et à l'impact de projets de tours alors naissants.

En 2001, alors que les études préalables au London Plan sont lancées, les propositions pour une stratégie de développement spatial font cas des immeubles de grande hauteur. Ils s'inscrivent alors dans le cadre de la "promotion d'une ville verte" respectueuse des préoccupations environnementales (GLA, 2001c, p. 13).

Dès lors les tours répondent officiellement à des attentes en termes de densification, d'environnement, et d'enrichissement urbanistique et esthétique reprenant l'argument d'une nécessaire qualité architecturale. Toutefois émerge parallèlement un autre discours où les tours ont également pour objectif de confirmer Londres dans son rôle de «world city» (GLA, 2001c, p. 7). Le bureau d'étude DEGW, commissionné par le GLA, confirme cette vision et souligne l'importance des tours en terme d'image (2002). Le mot « tour » est assumé et le bâti vertical constitue ainsi une sérieuse contribution à la promotion de Londres et à son statut. Le renouveau des tours peut alors s'opérer.

Figure 2.

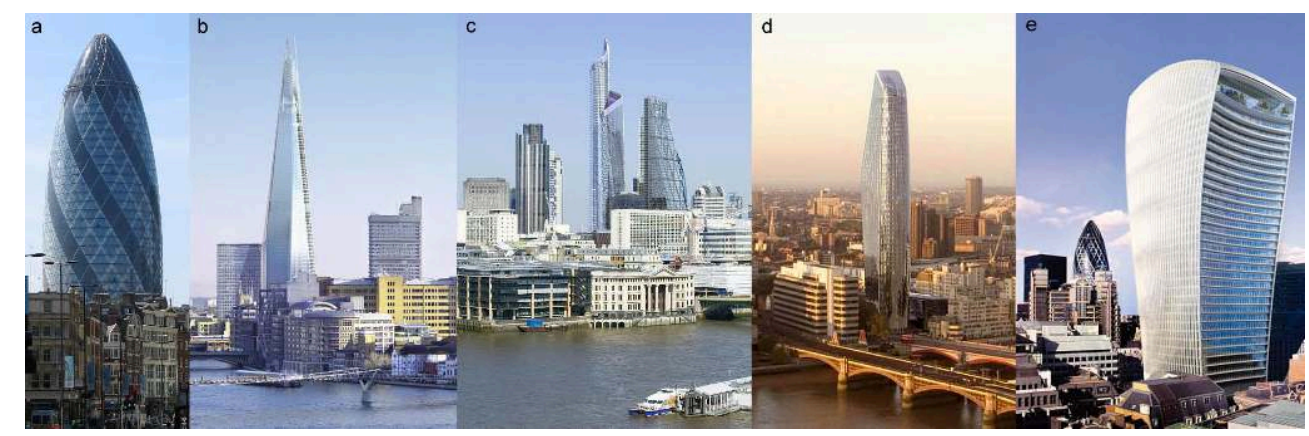

a) 30 st Mary Axe, 180 m, Sir Norman Foster, 2004.

b) London Bridge Tower, 306 m, Renzo Piano, 2011.

c) City, au centre Bishopsgate Tower, 288 m, KPF, 2011, à droite, Leadenhall Building, 225 m, Sir

Richard Rogers, 2010.

d) Beetham Tower, 220 m (N.B. : le projet a été réduit à 163 m), lan Simpson, 2012.

e) 20 Fenchurch, 160 m, Rafael Viñoly, 2011. 


\section{Les prémices du renouveau}

22 Le statut de Londres "ville monde " et l'image de la capitale britannique ne sont réellement promus à travers projets et réalisations que tardivement. Il fallut attendre que la réflexion sur l'urbanisme vertical se concrétise. Deux projets inaugurèrent une longue série à venir.

23 Au numéro 30 de St Mary Axe, le projet de Millenium Tower abandonné, le site est mis en vente. La compagnie d'assurance Swiss Re rachète le terrain et y envisage quelques mois plus tard la construction d'une tour, elle aussi signée de Lord Norman Foster. Le dessin est audacieux; une forme oblongue haute de 180 mètres couverte d'une résille bicolore (fig.2a). «La forme distincte de cette tour de 40 étages s'ajoutera à la grappe de tours qui symbolise le cour du centre financier de Londres $\|^{6}$ (Foster). C'est ainsi que l'architecte définit son projet lui conférant un rôle symbolique. Néanmoins dans le but de faire accepter l'ensemble, ce sont les considérations environnementales qui sont mises en exergue. Avancée technologique, la tour doit sa forme à une prise en compte des vents et appels d'air. En tant que tel, le projet reçoit l'approbation et le soutien du gouvernement, du maire de Londres, et du maire de la City.

Les travaux achevés en 2004, la tour est désormais visible depuis la Tamise, comme depuis la Tour de Londres. L'édifice reçoit à l'exceptionnelle unanimité le Stirling Prize, délivré alors depuis neuf ans par l'Institut Royal d'Architecture Britannique (RIBA) qui souhaite ainsi récompenser un édifice qui contribue amplement à l'histoire de l'architecture britannique. Lord Norman Foster recevant l'honneur de cette distinction, affirme avoir développé « une architecture écologique, la première du genre à Londres, qui, sans doute, par son indiscutable qualité, sera une référence dans la génération émergente des immeubles de grande hauteur $\aleph^{7}$ (Foster). Les Londoniens, quant à eux, et malgré quelques polémiques, se sont appropriés l'édifice en l'affublant de quelques sobriquets dont le " cornichon érotique ».

En 2000, naît également le projet de la London Bridge Tower. Renzo Piano, son architecte, propose une tour de 310 mètres, sur la rive sud de la Tamise, à Southwark (fig.2b). Le projet reçoit le soutien du maire ainsi que du Southwark Borough Council qui y voient l'objet d'une régénération urbaine de grande ampleur impliquant la modernisation de la London Bridge Station. A l'inverse, English Heritage, Historic Royal Palaces et les autorités de la Cathédrale Saint Paul s'opposent au projet clamant la défiguration de la ligne d'horizon et des perspectives depuis la Tour de Londres ou vers Saint Paul. Le gouvernement en la personne du Premier Ministre se prononce en faveur de la construction. A ce jour les travaux viennent de débuter.

De ces deux projets, qui marquent parallèlement la réflexion alors menée par le GLA, nous retenons d'une part le rôle d'un édifice qui s'impose comme un symbole pour Londres, et d'autre part un projet majeur, aux proportions inédites pour la capitale anglaise, qui pose de nouveau la question des vues et de la skyline, entre défiguration et amélioration. Le thème de l'image se fait alors sous-jacent ; image d'une ville à travers de nouveaux symboles urbains intégrés dans un paysage existant, reconnu et approprié, image de la ville à travers la prise en considération des vues et de la ligne d'horizon. Au delà, les tours sont argumentées comme les éléments moteurs de régénération urbaine et se présentent comme les outils nécessaires au développement urbain. 
Figure 3. 2012, vertical : projets et choix stratégiques

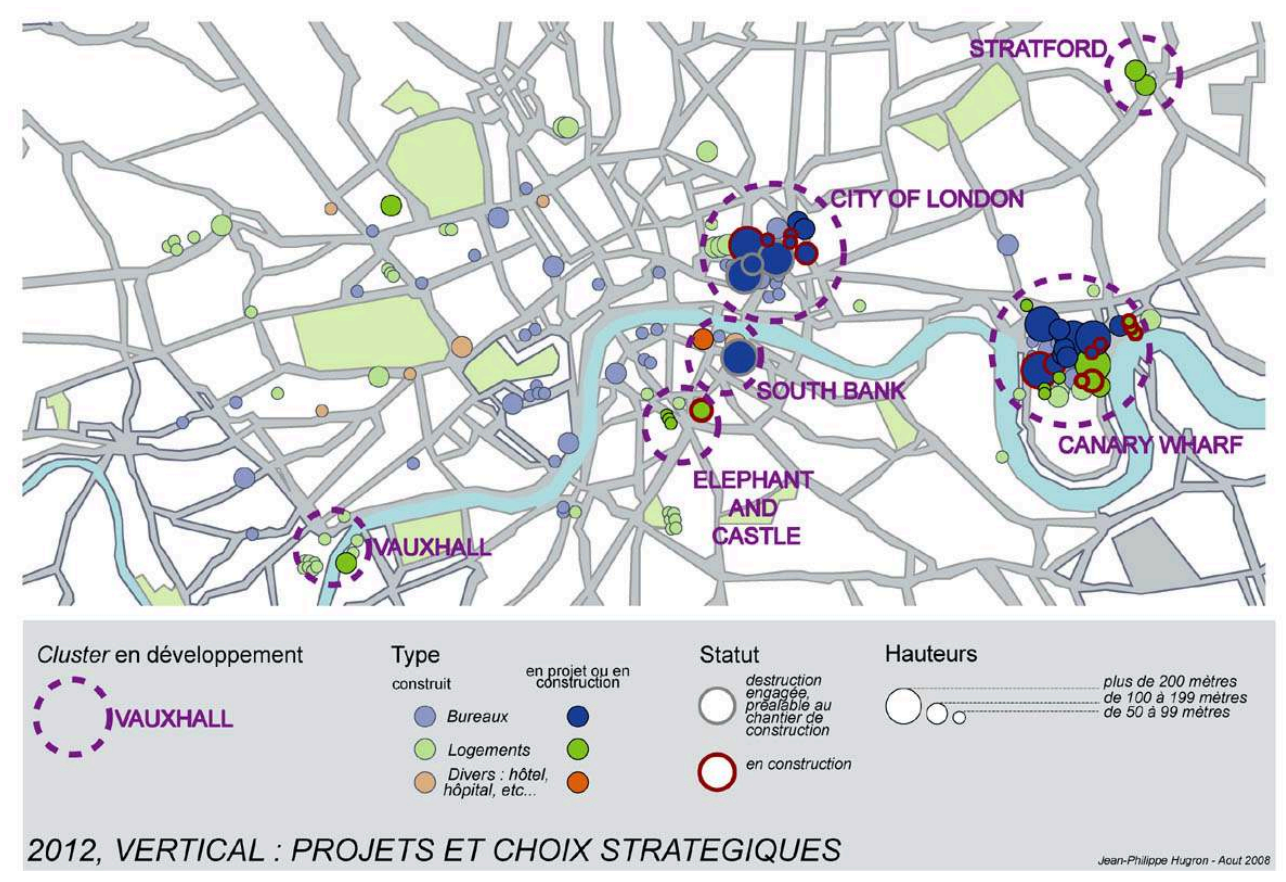

\section{Architecture verticale à Londres : un essai de typologie}

La construction verticale connaît un renouveau à Londres depuis l'arrivée de Ken Livingstone au poste de maire. Canary Wharf et la City concentrent les projets les plus prestigieux. Stratford, Elephant \& Castle, South Bank, Vauxhall sont privilégiés pour accueillir des grappes (cluster) de tours (cf. fig. 3).

Les dernières élections ont porté à la tête de la ville Boris Johnson, infligeant une défaite au maire sortant. Les tours comptèrent parmi les sujets de discorde. Quand l'un menait une politique décomplexée, l'autre dénonce les excès de son prédécesseur et prône quelques sites où la construction en hauteur serait circonscrite. La localisation des tours est donc un enjeu politique.

Toutefois l'apparente homogénéité que recouvre le thème de la verticalité, notamment à travers les débats passionnés entre partisans et adversaires de la hauteur ne laissent entendre aucun des différents processus matérialisés par projets et réalisations. Pourtant, l'observation du cas londonien démontre qu'il existe une diversité de tours comme de projets.

30 A l'échelle de la ville, l'érection d'immeubles de grande hauteur répond tant à des stratégies d'organisations spatiales comme la maximisation du développement des nœuds de transports ou l'amélioration de l'espace public qu'à une stratégie de communication afin de promouvoir le statut de Londres ville monde. En revanche à l'échelle de la parcelle ou du bâti, les motivations, différentes, sont nuancées.

31 Ici motivée par un processus de régénération, là par la modernisation d'un patrimoine obsolète, les tours ne peuvent s'envisager de façon générale. Définir une typologie des tours à Londres est dès lors nécessaire afin de comprendre un phénomène trop souvent perçu comme homogène. 

King's Reach Tower. L'édifice haut de 111 mètres, dessiné par Seifert and Partners auteurs de la célèbre Tower 42, est aujourd'hui obsolète. Les aménagements de 1972 vieillissent et ne font que souligner l'échec urbanistique du projet, notamment du Milroy Walk, centre commercial au succès timide. Propriété de Capital and Counties, ces derniers imaginent remodeler la tour. En faisant appel à l'architecte Ken Shuttleworth, connu pour son trait audacieux, longtemps exercé au sein de l'agence Foster and Partners, le promoteur ambitionne un projet remarquable. La forme de la tour est préservée, son revêtement modernisé. Derrière quelques couleurs criantes, 
azur et blanche, il s'agit avant tout d'offrir un confort thermique. Tant l'isolation que la ventilation sont pensées pour répondre aux exigences environnementales actuelles. $\mathrm{Au}$-delà, c'est un projet de régénération qui est entrepris. Né d'une étroite concertation avec les usagers du site, Ken Shuttleworth imagine un « office village ». La base de la tour est retravaillée ; plusieurs petits édifices, de même facture, viennent s'agglomérer au pied de la tour. Magasins et services y trouveront place. L'ambition est de créer un « espace public accueillant et vivant ». Le projet approuvé n'est pour autant pas encore sorti de terre. La tour est rachetée en février 2007 par le magnat syrien Simon Halabi alors à la tête de la compagnie Buckingham Securities. Les plans de Shuttleworth sont abandonnés; un nouveau cabinet, Hamiltons Architects, est appelé à repenser le développement de cette tour.

Le cas de la King's Reach Tower illustre l'enchevêtrement des processus de régénération et de réhabilitation. Toutefois un nombre important de tours sont à même de subir des transformations plus ou moins profondes sans qu'il ne s'agisse de les inclure dans le cadre plus vaste d'une régénération. La plupart des tours construites à Londres furent érigées lors des trois décennies suivant l'achèvement du second conflit mondial. Elles représentent un parc immobilier vieillissant sinon obsolète. Energivores, elles sont coûteuses en charges, irrespectueuses des normes environnementales, et deviennent inefficaces quant à leurs aménagements. Aussi la plupart des tours de la City et plus largement de Central London connaissent d'importants travaux allant de simples réaménagements d'espaces intérieurs jusqu'au remodelage complet de l'édifice. Ces travaux de réhabilitation opèrent une mise aux normes du bâti et sa modernisation.

Propriétaires, promoteurs et architectes s'accordent alors pour trouver les solutions nécessaires. Dans le cas de la King's Reach Tower, Capital and Counties et Ken Shuttleworth imaginèrent une restructuration profonde touchant, dénaturant pour certains, l'image de la tour construite par Seifert. Dans d'autres cas, la spécificité architecturale de l'édifice est préservée. A l'instar de Centre Point, ces tours ne connaissent que des réaménagements internes. Aussi en 2002, les architectes Gaunt Francis Associates opérèrent une réhabilitation respectueuse des parties extérieures. English Heritage, soucieux de la préservation de l'architecture originale de la tour, observa de près les travaux et travailla même avec les architectes. En effet l'architecture de Centre Point, pensée elle aussi par Seifert, fut en 1994 classée au grade $\mathrm{II}^{9}$, et ce après deux tentatives infructueuses.

41 Les polygones de béton de Centre Point eurent plus de chances que ceux de Stock Exchange Tower. La tour brutaliste de la City est la cible d'une profonde restructuration, aujourd'hui en cours d'achèvement. Grimshaw and Partners ont coiffé la tour de deux étages supplémentaires et ont entièrement métamorphosé les façades en leur apposant un revêtement de verre. Un pan du patrimoine est aujourd'hui menacé par les exigences accrues du marché.

\section{Réhabiliter autrement : reconvertir}

Les tours évoquées jusqu'à présent sont des tours de bureaux qui ont conservé ou conservent leur vocation première. Toutefois certains projets de réhabilitation se sont accompagnés d'une reconversion. Parmi ces édifices, Marathon House. La tour de 16 étages imaginée en 1955, livrée en 1960, abritait la compagnie Castrol qui est devenue par la suite Marathon Oil. La vente de l'immeuble fut signée dans les années quatre- 
vingt-dix. L'architecte Leighton Carr envisagea la reconversion de l'immeuble. Les bureaux ont alors été réaménagés en appartements "retro-chic» célébrant le modernisme des sixties. L'initiative était pour lors inédite.

Un même destin fut accordé à l'Alexander Fleming House. La tour de 55 mètres imaginée par Ernö Goldfinger abrita le ministère de la santé. Alors que l'immeuble est abandonné, le promoteur St George décida d'en faire l'acquisition. En 1997, il livre, après d'importants travaux préservant l'architecture extérieure, une résidence de logements de luxe.

La reconversion ne concerne pas uniquement des immeubles de bureaux transformés en logements. Aragon Tower, tour de logements sociaux, haute de 91 mètres connut une audacieuse reconversion. Alors que le Borough de Lewisham ne peut engager l'importante réhabilitation qu'elle ambitionne, elle décide pour trouver les fonds nécessaires de vendre l'un des immeubles de l'ensemble. Aragon Tower est acheté en 1999 par Berkeley Homes au prix de 10,5 millions de livres. Le promoteur décide de réaménager la tour en logements de standing et d'y ajouter cinq nouveaux étages abritant de luxueux penthouses. La réalisation de ce projet permit au quartier de se doter d'une nouvelle mixité sociale qui lui faisait jusqu'alors défaut.

\section{Rénovation : détruire pour reconstruire}

Si certaines tours sont réaménagées restructurées ou reconverties, d'autres sont détruites. Une partie du parc immobilier, obsolète, constitue de véritables opportunités foncières. Il s'agit alors au propriétaire et au promoteur de raser l'ancien édifice pour en proposer un autre. Nous avons d'ores et déjà observé ce processus dans le cas de la London Bridge Tower où l'ensemble des Southwark Towers est démoli dans la perspective du projet de Renzo Piano. Au cœur de la City, 20 Fenchurch, propriété de Land Securities, ou encore P\&O Building, propriété de British Land, sont en cours de destruction. Chacune de ces tours sera à terme remplacée par un édifice plus haut, respectivement par le Talkie Walkie, immeuble dessiné par Rafael Viñoly et par le Leadenhall Building de Richard Rogers. Ces projets ont obtenu l'ensemble des autorisations nécessaires, et ce, non sans difficulté. English Heritage émit de vives critiques, rappelant l'obligatoire préservation des vues et perspectives vers St Paul. Aussi les projets furent modifiés afin de satisfaire ces attentes particulières.

46 A quelques encablures, une autre tour de Seifert, Drapers Gardens connaît un sort différent. Une vaste entreprise de rénovation est planifiée par The Royal Bank of Scotland Group. La tour combinant élégamment béton et vitres fumées ne propose pas de plateaux suffisamment grands. Une restructuration est alors inenvisageable. La destruction de l'édifice est alors programmée. Les 16710 mètres carrés, répartis sur une hauteur de 110 mètres, seront à terme remplacés par un groundscraper, immeuble de bureaux aux proportions massives, offrant 39131 mètres carré pour une hauteur de 75 mètres. L'annonce de la destruction de la tour provoqua quelques réactions. La Twentieth Century Society milita pour sa préservation, en vain. English Heritage de son côté se satisfit de la disparition d'une structure qui entravait les perspectives visuelles en direction de Saint Paul. Aujourd'hui Drapers Gardens détruit, le nouvel immeuble est en chantier.

47 Les rénovations s'opèrent généralement sur des sites stratégiques. La destruction d'une tour reste un événement marginal. Il s'agit généralement pour le propriétaire et le 
promoteur de maximiser un foncier alors occupé par une construction inadaptée au marché actuel. Une réhabilitation offrirait un édifice modernisé, mais les choix stratégiques portent à la création d'édifices plus grands, en hauteur et/ou en surface.

\section{E. Patrimonialisation}

48 Véritables opportunités, les tours vieillissantes sont la cible de transformation ou de destruction. Restructurées, réhabilitées, rénovées ou reconverties, ces architectures modernistes, brutalistes, de style international, sont un patrimoine aujourd'hui menacé. Drapers Gardens fut démoli sans que la Twentieth Century Society ne puisse agir. A l'inverse, en étant répertorié au grade II, Centre Point fut préservé.

Lister un édifice équivaut à la reconnaissance de son caractère original ou de son intérêt historique. Selon le grade accordé, des aides à la modernisation ou à la restauration peuvent être accordées par English Heritage. Enfin toute modification portée sur un édifice doit être soumise à l'avis des autorités locales. Une liste des travaux autorisés, nécessitant ou non approbation (LBC, Listed Building Consent) est dressée. L'objectif est de préserver ainsi un bâti remarquable de toute altération qui pourrait porter atteinte au caractère reconnu.

Certaines tours sont aujourd'hui classées, et leur valeur architecturale est ainsi avérée. En 2001, le complexe résidentiel de Barbican fut, à l'instar de Centre Point, répertorié au grade II. Les trois tours ainsi que l'ensemble des infrastructures du quartier sont désormais préservés. Cette décision fut motivée par l'intérêt suscité par le site. Le ministre d'Etat aux Arts, Tessa Blackstone, souligna tant le langage architectural adopté, que la composition formelle, la structure, les matériaux, ou encore le parti pris urbanistique associant tours et dalle.

51 Répertorier ces architectures est une étape nécessaire, néanmoins, dans l'imaginaire collectif, ces réalisations n'ont que peu de valeur. La transformation d'une tour ou sa disparition ne suscite pas encore l'émoi escompté par quelques associations militantes.

A l'inverse, les nouveaux projets tendent à susciter l'enthousiasme. Canary Wharf et la City vont connaître, à terme, de spectaculaires changements (cf. fig. 4). Les rares et extraordinaires rénovations imposant la destruction de tours comme préalable à toute nouvelle construction ne s'opèrent qu'au cœur de la puissante place financière, voire à ses portes. L'attractivité de la City oblige également propriétaires et promoteurs à moderniser un patrimoine vieillissant. Aussi il n'est pas étonnant que les réhabilitations soient plus nombreuses à mesure que l'on s'approche du cœur économique de la capitale. Cette géographie confirme la prédominance de la City et de Canary Wharf et souligne la transformation des espaces alentours entre modernisation et régénération. 
Figure 4. Architecture verticale : une variété de projets et de réalisations

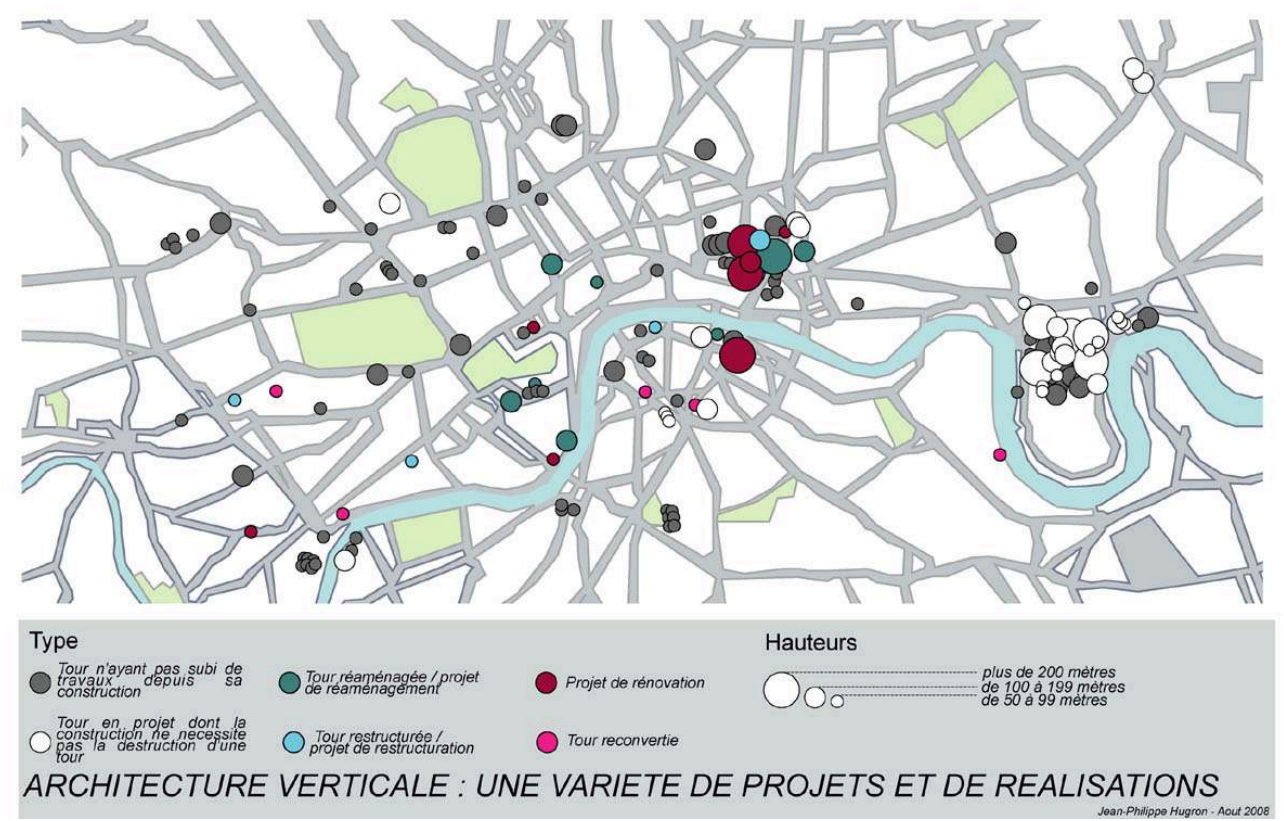

\section{Conclusion}

L'expérience moderniste des tours avait laissé quelques traumatismes. L'adage « form follow function» fut a posteriori repris et ironiquement reformulé «form follow fiasco ». Une politique d'endiguement (containment) de la hauteur interdit toute construction de tour en dehors d'un nouveau périmètre, constitué ex-nihilo ; Canary Wharf est alors amené à concentrer l'ensemble des constructions verticales à venir, régénérant de fait un des espaces les plus déshérités de la capitale britannique. Quelques timides projets au cours des années quatre-vingt-dix ne purent aboutir. Parallèlement une partie des tours héritées de l'après-guerre subit des travaux de réhabilitation. Les années 2000 virent s'amorcer, avec la construction du 30 st Mary Axe, le cornichon érotique, un mouvement de confiance. Le succès médiatique de l'architecture imaginée par Norman Foster fit des émules. Aussi l'élaboration du London Plan intègre une politique incitatrice. Les tours sont envisagées comme autant de solutions aux problèmes urbains et se présentent comme les outils, sinon comme les moteurs de vastes régénérations. Ce renouveau de l'architecture verticale se concrétise aujourd'hui sous diverses formes. Réhabilitation, restructuration, réaménagement, reconversion, rénovation, patrimonialisation engendrent une strate de réalisations nouvelles. Il est donc impossible d'appréhender les tours, type architectural, comme un simple objet homogène. La typologie ici proposée s'offre comme le préalable à toute étude géographique d'un phénomène majeur de l'urbanisme londonien contemporain. 


\section{BIBLIOGRAPHIE}

CABE, English Heritage (2007). - Guidance on tall buildings, Londres, CABE, 7 p.

DEGW (2002). -London's skyline, views and high buildings, Londres, DEGW, 114 p.

English Heritage (2004). -Barbican, Listed Building Management Guidelines, vol. 1, Londres, English Heritage, $39 \mathrm{p}$.

English Heritage (2004). -Barbican, Listed Building Management Guidelines, vol. 2, Londres, English Heritage, $81 \mathrm{p}$.

FRAMPTON K. (2007). - “Architecture in the age of Globalization”. In :Visionary Power, producing the contemporary city, Rotterdam, NAI, p. 131-171.

GLA (2001a). - Interim strategic planning guidance on tall buildings, strategic views and the skyline in London, Londres, GLA, 19 p.

GLA (2001b). - Summary: Towards the London Plan, Londres, GLA, $20 \mathrm{p}$.

GLA (2001c). - Towards the London Plan, Londres, GLA, 114 p.

GLA (2002). -Responses to towards the London Plan, Londres, GLA, 37 p.

GLA (2004a). - "The crosscutting policies". In : The London Plan, Londres, GLA, p. 55-219.

GLA (2004b). - The London Plan : a summary, Londres, GLA, 36 p.

GLA (2005). -DIFA Tower Planning Report, Londres, GLA, 14 p.

GLA (2006). -20 Fenchurch Planning Report, Londres, GLA, 13 p.

GOTTMANN J. (1966). - “Why the skyscraper?”, Geographical Review, p. 190-212.

HALL R. -Built Identity: Swiss Re's Corporate Architecture, Basel, Birkhauser Verlag AG, 200 p.

HALL T. (1998). - The entrepreneurial city : geographies of politics, regime, and representation, NewYork, John Wiley \& Sons, 370 p.

JENCKS C. (2005). - The iconic building, Londres, Frances Lincoln, 224 p.

MORAND P. (1962). - «Le nouveau Londres ». In : Morand P. (2001), Voyages, Paris, Robert Laffont, Bouquins, p. 485-576.

ROGERS R. (2000). - Proof of evidence, Londres, GLA, 33 p.

ROGERS R. (2000). - My vision for London's urban renaissance, Londres, Mayor of London, $12 \mathrm{p}$.

SHORT M. (2004). - "Regulating the impact of proposals for new tall buildings on the built heritage", Planning History, Vol. 26, n³, p. 3-10.

SHORT M. (2005). - UK Tower Proposals - Tall Buildings, http://www.e-architect.co.uk/

SIMON R. D. (1996). - "Skyscrapers and the New London Skyline : 1945-1991", The Electronic Journal of Architecture, http://architronic.saed.kent.edu/

Urban Task Force (1999). - Toward an urban Renaissance, Londres, UTF, 20 p.

WRIGHt H. (2006). -London high, Londres, Frances Lincoln, 232 p.

Sites internet : 
20 Fenchurch Street : http://www.20fenchurchstreet.co.uk

British Land (Promoteur) : http://www.britishland.com

Centre Point : http://www.centrepoint-london.com

City of London Corporation : http://www.cityoflondon.gov.uk

Drapers'Gardens : http://www.drapersgardens.com

English Heritage : http://www.english-heritage.org.uk

Foster and Partners : http://www.fosterandpartners.com

GLA (Greater London Authority) : http://www.london.gov.uk

Land Securities (Promoteur) : http://www.landsecurities.com

London Bridge Tower : http://www.shardlondonbridge.com

Make Architects - Ken Schuttleworth : http://www.makearchitects.com

NLA (New London Architecture) : http://www.newlondonarchitecture.org

RIBA : http://www.architecture.com

Rogers Stirk Harbour + Partners : http://www.rsh-p.com

Skyscrapernews : http://www.skyscrapernews.com

Twentieth Century Society : http://www.c20society.org.uk

World Architecture News : http://www.worldarchitecturenews.com

\section{NOTES}

1. "Visual intrusion, location, site size, over-shadowing, local character, effects on the river Thames and open space, architectural quality and night scene."

2. Richard Rogers (1933) est architecte diplômé de l'Université de Yale. Il forme une première agence avec Norman Foster en 1963. Ensemble et dans un contexte d'architecture prospectiviste, ils orientent leur recherche vers les considérations fonctionnalistes et technologiques. Toutefois c'est avec Renzo Piano qu'ils remportent la réalisation du centre culturel du plateau Beaubourg. En 1977, il fonde sa propre agence, Richard Rogers Partnership. Fidèle à ses convictions architecturales, il réalise le siège de la Lloyds à Londres qui aujourd'hui illustre les débuts d'un courant dit « high tech».

Rogers reçoit de nombreuses distinctions, la médaille d'or de l'Institut Royal d'Architecture Britannique en 1985 ou encore le Pritzker Price en 2007. En 2006 il reçoit également le Lion d'Or de la Biennale d'architecture de Venise sur le thème "Villes, architecture et société", prix qui récompense son travail et sa réflexion sur les villes.

3. L'UTF regroupe des personnalités telles que Richard Burdett, professeur d'architecture et d'urbanisme, Sir Peter Hall, professeur d'aménagement, Anne Power, professeur en politique sociale, Sir Crispin Tickell, chancelier de l'université du Kent,...

4. "I support high buildings, both as clusters (such as in the City, Canary Wharf and Croydon), and as stand-alone buildings (such as the Post Office Tower and Millbank Tower), where they are in close proximity to a major public transport interchanges and contribute to the quality of London's environment. I have no objection in principle to London having the tallest of buildings." 
5. Le mot tower n'est que très rarement utilisé en anglais pour désigner une construction verticale abritant des logements ou quelques activités économiques. Le mot skyscraper (gratteciel) est teinté d'américanisme, l'expression building qui éveille l'image de la verticalité pour un Français, ne relève que de la construction pour un Anglais. L'expression «tall building ", plus neutre, s'apparenterait de prime abord à « l'immeuble de grande hauteur » (I.G.H.). Néanmoins cette dernière notion recouvre en français une législation que ne porte pas la proposition anglaise. Aussi la traduction la plus fidèle serait "immeuble-tour», expression largement diffusée par les revues françaises d'architecture jusqu'au milieu des années 70 mais qui est restée inutilisée dans le langage courant. Aussi le mot «tour» apparaît comme le plus fidèle pour traduire « tall building ».

6. "The distinctive form of the 40-storey tower will add to the cluster of tall buildings that symbolise[s] the heart of London's financial centre.» (d'après Short (2005), lu sur le site www.fosterandpartners.com le 8 octobre 2004.

7. "Developed on the precedents of green architecture for which the practice is renowned, the Foster designed 30 st Mary Axe is the capitals first ecologically progressive skyscraper, and its uncompromising quality has set a benchmark for an emerging generation of tall buildings." Lu sur le site http://www.fosterandpartners.com le 11 septembre 2007.

8. "Tall buildings will be supported where they create attractive landmarks enhancing London's character, help to provide a coherent location for economic clusters of related activities or act as a catalyst for regeneration."

9. English Heritage, aussi connu sous le nom de Historic Buildings and Monuments Commission for England, est en charge de protéger et de promouvoir le patrimoine anglais. Les édifices sont classés selon le grade que la commission leur a attribué. Le grade I honore les édifices « d'intérêt exceptionnel » (2\%), le grade II*, les édifices « d'intérêt particulier » $(4 \%)$, le grade II, les édifices « d'intérêt particulier dont il faut garantir tous les efforts pour leur sauvegarde » (94\%).

\section{RÉSUMÉS}

L'architecture verticale connaît un nouvel élan dans la capitale britannique. Enjeux de vastes projets de régénération urbaine, les tours servent aussi l'image de la ville. Derrière l'apparente homogénéité d'un type architectural, les différentes stratégies optées laisse apparaître une variété de processus. Une typologie des tours est alors un préalable à toute étude géographique. Réhabilitation, rénovation, reconversion, patrimonialisation consacrent le renouveau des tours à Londres.

Skyscrapers are booming in London. Tall buildings act as a catalyst to improve the quality of the urban environment and provide significant regeneration enhancing the image of the city. As an architectural type, tall buildings respond to different strategies. A typology is required to develop any geographical studies. Refurbishing, remodeling, renovation, conversion, protection contribute to the skyscrapers renewal in London

Die Wolkenkratzer erleben einen neuen Aufschwung in London. Hochhaüser spielen eine entscheidende Rolle nicht nur für die Regenerierung der Stadt, sondern auch für das Stadtbild. Verschiedene Strategien verstecken sich hinter der scheinbaren Homogenität eines Baustils. Eine 
Geografie der senkrechten Architektur benötigt zunächst eine Typologie. Sanierung, Renovierung, Umstellung, Schutz, verankern die Erneuerung der Hochhaüser in London.

INDEX

Mots-clés : architecture verticale, Londres, régénération, réhabilitation, rénovation, tour Schlüsselwörter : Erneurung, Hochhaüser, London, Renovierung, Sanierung

Keywords : London, regeneration, renewal, skyscrapers, tall building

\section{AUTEUR}

\section{JEAN-PHILIPPE HUGRON}

Doctorant, Institut d'Urbanisme de Paris XII, GERPHAU/UMR LOUEST,jphh@wanadoo.fr 